\title{
ON THE FIRST ORDER THEORY OF THE ARITHMETICAL DEGREES
}

\author{
PIERGIORGIO ODIFREDDI
}

\begin{abstract}
The first order theory of the arithmetical degrees with arithmetical jump is not elementarily equivalent to the first order theory of the Turing degrees with jump.
\end{abstract}

1. The result and some related problems. The arithmetical degrees are equivalence classes with respect to the equivalence relation: $A \equiv{ }_{a} B$ iff $A \leqslant{ }_{a} B$ and $B \leqslant{ }_{a} A$, where $A \leqslant{ }_{a} B$ means that $A$ is arithmetical in $B$ (i.e. definable from $B$ in an arithmetical way). By $\mathscr{D}_{a}$ we mean the structure of the arithmetical degrees with the partial ordering induced by $\leqslant_{a}$ on the equivalence classes. By $\mathscr{D}_{a}^{\prime}$ we mean $\mathscr{D}_{a}$ with the unary operation (called arithmetical jump) induced by the $\omega$-jump (Rogers [5, p. 258]) on the equivalence classes. Some results about the structures $\mathscr{D}_{a}$ and $\mathscr{D}_{a}^{\prime}$ are collected in our paper [4] (to which we refer for notations, unexplained terminology and background). These structures have been less studied than the structures of the Turing degrees with or without jump ( $(D)$ and $~(D)$, respectively). Harding [2] has been able to show (by using initial segments embeddings, hence by quite sophisticated methods) that $\mathscr{D}_{a}^{\prime}$ and $\mathscr{D}^{\prime}$ are not isomorphic. The purpose of this note is to prove the following:

THEOREM. 'Q ${ }_{a}^{\prime}$ is not elementarily equivalent to 'D'.

The proof is simple and does not require new methods beyond those introduced in Sacks [6]. Nevertheless we think the result is a step toward a better understanding of the arithmetical jump, a seemingly refractary object to study. An apparently difficult problem about it is the following:

Problem 1. Determine the range of the arithmetical jump operator restricted to the arithmetical degrees below $\mathbf{0}^{\prime}$.

The referee has noted that not every arithmetical degree between $\mathbf{0}^{\prime}$ and $\mathbf{0}^{\prime \prime}$ is the jump of an arithmetical degree below $\mathbf{0}^{\prime}$, since if $A \leqslant{ }_{a} 0^{\omega}$ then $A^{\omega}$ is an arithmetical singleton, but there exists $B$ such that $0^{\omega} \leqslant{ }_{a} B \leqslant{ }_{a} 0^{\omega+\omega}$ and $B$ is not an arithmetical singleton. We thus propose the following:

Conjecture. If $\mathbf{a}<\mathbf{0}^{\prime}$ then $\mathbf{a}^{\prime}=\mathbf{0}^{\prime}$.

Received by the editors January 2. 1982.

1980 Mathematics Subject Classification. Primary 03D30.

Key words and phrases. Arithmetical degree, arithmetical jump, arithmetically pointed tree. local forcing.

C 1983 American Mathematical Society $0002.9939 / 82 / 0000-0453 / \$ 01.75$ 
This would imply the Theorem of this note, since the analogous fact fails for Turing degrees; see Shoenfield [7].

It may be worth noting that for the structure $\mathscr{D}_{h}^{\prime}$ of hyperdegrees with hyperjump (see Rogers [5, Chapter 16]) the analogous fact holds (Spector [9]): this shows $\mathscr{O D}_{h}^{\prime} \not \equiv \mathcal{Q}^{\prime}$. That $\mathscr{D}_{h}^{\prime} \not \equiv \mathscr{D}_{a}^{\prime}$ follows from the fact that the smallest ideal closed under jump has least upper bound in $\mathscr{D}_{h}^{\prime}$ but not in $\mathscr{D}_{a}^{\prime}$.

A solution to the following problem might also shed light on the nature of the arithmetical jump operator.

Problem 2. Are the arithmetical degrees below $\mathbf{0}^{\prime}$ and the Turing degrees below $\mathbf{0}^{\omega}$ elementarily equivalent?

Note that the sets involved here are respectively those arithmetical and recursive in $0^{\omega}$.

A natural question concerns the extension of the elementary inequivalence proved here to the structures without jump; in particular,

Problem 3. Is $\mathscr{D}_{a}$ elementarily equivalent to $\mathscr{Q}$ ?

With respect to the similarly open questions:

Is $\mathcal{D}_{h}$ elementarily equivalent to ${ }^{\circ} D_{a}$ ?

Is $\mathcal{O D}_{h}$ elementarily equivalent to $\mathcal{O}$ ?

It is at least known that the negative answers to them are consistent with ZFC. Indeed Simpson [8] has proved that if $V=L$ then there is no cone of minimal covers of hyperdegrees, while such cones are known to exist for both Turing degrees and arithmetical degrees (by Borel determinacy and a lemma of Martin [3]).

2. The proof. Let $I$ be an ideal: we say that a is a 1-least upper bound (1-1.u.b.) for $I$ if it is the least element of $\left\{\mathbf{c}^{\prime}:(\forall \mathbf{b} \in I)(\mathbf{b} \leqslant \mathbf{c})\right\}$. Sacks [6] has proved that in ' $2 \mathbf{D}^{\prime}$ the smallest jump-ideal has no 1-1.u.b.. It is enough to show that in $\mathscr{Q}_{a}^{\prime}$ the smallest jump-ideal has 1-1.u.b. (Note that "the smallest jump-ideal has 1-1.u.b." is indeed expressible as a first order sentence in both $\mathrm{O}^{\prime}$ ' and $\left.{ }^{\circ}\right)_{a}^{\prime}$, because in both every countable ideal has an exact pair. So quantification over ideals can be replaced by quantification over pairs of degrees. See [4] for details.)

Since some confusion might arise between arithmetical degrees and Turing degrees, we recall (see [4]) some definitions:

$\mathbf{0}^{n}=$ the arithmetical degree of $0^{\omega \cdot n}$ (remember that the arithmetical jump is induced by the $\omega$-jump operator);

$\mathbf{0}^{\omega}=$ the arithmetical degree of $0^{\omega^{2}}$.

The smallest jump-ideal of ' $\left.{ }^{\prime}\right)_{a}^{\prime}$ is $I=\left\{\mathbf{b}:(\exists n)\left(\mathbf{b} \leqslant \mathbf{0}^{n}\right)\right\}$. We show that $\mathbf{0}^{\omega}=1-1$. u.b. of $I$.

First note that if $(\forall n)\left(0^{\omega \cdot n} \leqslant{ }_{a} A\right)$ then $0^{\omega^{2}} \leqslant{ }_{a} A^{\omega}$ (in terms of arithmetical degrees this says: if $(\forall n)\left(0^{n} \leqslant \mathbf{a}\right)$ then $\left.0^{\omega} \leqslant \mathbf{a}^{\prime}\right)$. Indeed, if $0^{\omega \cdot n} \leqslant{ }_{a} A$ then for some $m, 0^{\omega \cdot n} \leqslant{ }_{T} A^{m}$ and so $0^{\omega \cdot n} \leqslant{ }_{T} A^{\omega}$. By the Enderton-Putnam [1] computation, if this holds for every $n$ then $0^{\omega^{2}} \leqslant{ }_{T} A^{\omega+2}$ and so $0^{\omega^{2}} \leqslant{ }_{a} A^{\omega}$.

It is now enough to show that there is $B$ such that $(\forall n)\left(0^{\omega \cdot n} \leqslant{ }_{a} B\right)$ and $B^{\omega} \leqslant{ }_{a} 0^{\omega^{2}}$ (in terms of arithmetical degrees this says that $(\forall n)\left(\mathbf{0}^{n} \leqslant \mathbf{b}\right)$ and $\left.\mathbf{b}^{\prime} \leqslant \mathbf{0}^{\omega}\right)$. Note that we cannot get $B$ such that $(\forall n)\left(0^{\omega \cdot n} \leqslant{ }_{T} B\right)$ since otherwise (by the same computation above) $0^{\omega^{2}} \leqslant{ }_{T} B^{2}$ and so $B^{\omega} \leqslant{ }_{a} B$. We build a sequence $\left\{T_{n}\right\}_{n \in \omega}$ of arithmetically pointed trees (i.e. trees arithmetical in every one of their branches) of 
arithmetical degree $0^{n}$. Two basic lemmas from Sacks [6] are:

(1) If $P$ is arithmetically pointed, $Q \subseteq P$ and $Q \leqslant{ }_{a} P$, then $Q$ is arithmetically pointed and $Q \equiv{ }_{a} P$.

(2) If $P$ is arithmetically pointed and $P \leqslant{ }_{a} A$, then there is an arithmetically pointed $Q \subseteq P$ such that $Q \equiv{ }_{a} A$.

We will have $B \in \cap_{n \in \omega} T_{n}$ and by pointedness this will ensure $(\forall n)\left(0^{\omega \cdot n} \leqslant{ }_{a} B\right)$. To get $B^{\omega} \leqslant{ }_{a} 0^{\omega^{2}}$ let $\left\{\varphi_{n}\right\}_{n \in \omega}$ be a recursive enumeration of the arithmetical sentences $n \in X^{\omega}$. Let $T_{0}=$ full binary tree. Given $T_{n}$ let $Q_{n} \subseteq T_{n}$ be a subtree of it of sufficiently generic sets, such that one of the following holds:

For every $A$ on $Q_{n}, A \vDash \varphi_{n}$.

For every $A$ on $Q_{n}, A \vDash \sim \varphi_{n}$.

Sufficiently generic means $m$-generic for the least $m$ such that $\varphi_{n} \in \Sigma_{m}^{0}$. $Q_{n}$ is built by local forcing on $T_{n}$ and is clearly possible to get it arithmetical in $T_{n}$ (since $\varphi_{n}$ is arithmetical). So by lemma (1), $Q_{n}$ is arithmetically pointed and $Q_{n} \equiv{ }_{a} T_{n}$. Let $T_{n+1} \subseteq Q_{n}$ be an arithmetically pointed tree of arithmetical degree $\mathbf{0}^{n+1}$ (by lemma (2)). The construction is clearly recursive in $0^{\omega^{2}}$, and so $B^{\omega} \leqslant{ }_{a} 0^{\omega^{2}}$ (actually $B^{\omega} \leqslant T$ $0^{\omega^{2}}$ ), because to decide if $n \in B^{\omega}$ it is enough to see whether for any branch $A$ of $T_{n+1}, A \vDash \varphi_{n}$. Choose $A$ recursive in $T_{n+1}$ (e.g. the leftmost branch) and see if $A \vDash \varphi_{n}$ : this is arithmetical in $A$, hence arithmetical in $T_{n+1}$ and thus recursive in $0^{\omega \cdot(n+2)} \leqslant{ }_{T} 0^{\omega^{2}}$ (uniformly in $n$ ).

\section{BIBLIOGRAPHY}

1. Enderton and Putnam, $A$ note on the hyperarithmetical hierarchy, J. Symbolic Logic 35 (1970), 429-430.

2. Harding, Forcing in recursion theor, $\mathrm{Ph}$. D. Thesis, University College of Swansea, 1974

3. Martin. The axiom of determinateness and reduction principles in the arithmetical hierarchy. Bull. Amer. Math. Soc. 74 (1968), 687-689.

4. P. Odifreddi, Forcing and reducibilities, J. Symbolic Logic 47 (1982).

5. Rogers, Theon of recursive functions and effective computabilit, McGraw-Hill, New York, 1967.

6. G. Sacks. Forcing with perfect closed sets, Proc. Sympos. Pure Math., vol. 13. Amer. Math. Soc., Providence, R. I.. 1971, pp. 331-355.

7. J. Shoenfield. On degrees of unsolvability. Ann. of Math. (2) 69 (1959), 644-653.

8. Simpson. Minimal covers and hyperdegrees. Trans. Amer. Math. Soc. 209 (1975), 45-64.

9. Spector, Recursive well-orderings, J. Symbolic Logic 20 (1955), 151-163.

Istituto Matematico. Universita di Torino. Torino, Italy 